\title{
The Comparison of Pelvic Mass Score and Risk of Malignancy Index-3 in Discrimination of Benign and Malignant Adnexal Masses
}

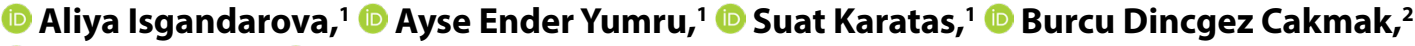 \\ (D) Betul Dundar, ${ }^{2}$ 이 Ulku Ayse Turker ${ }^{2}$
}

'Department of Obstetrics and Gynecology, University of Health Sciences Turkey, Sisli Hamidiye Etfal Teaching and Research Hospital, Istanbul, Turkey

${ }^{2}$ Department of Obstetrics and Gynecology, University of Health Sciences Turkey, Bursa Yuksek Ihtisas Research and Training Hospital, Bursa, Turkey

\begin{abstract}
Objectives: Discrimination of benign and malignant adnexal masses is crucial for the follow-up and prognosis of the patient. Since each modality alone does not have enough sensitivity, the combination of all methods called multimodal screening is currently being used. In this study, we aimed to compare pelvic mass score (PMS) and the risk of malignancy index (RMI-3) scoring systems in determining the malignant potential of adnexal masses.

Methods: In this prospective observational study, 40 patients between the age of 15-79 who were diagnosed as an adnexal mass were included between March and October 2016. Patients were classified as benign $(n=20)$ and malignant $(n=20)$. The age, gravida, parity, findings of a pelvic examination, medical and family history, laboratory parameters, sonographic findings, histopathological results, PMS and RMI-3 scores of the patients were recorded.

Results: The mean age, CA-125 levels, Sassone scores and ultrasonography scores of patients were higher in malignant cases, whereas the resistance index was lower. Both RMI-3 and PMS scores were higher in the malignant group (1728.14 \pm 325.3 vs. $36.27 \pm 31.01, p<0.001$ and $55.31 \pm 40.96$ vs. $9.91 \pm 5.29, p<0.001$, respectively). Receiver operating curve for the predictive value of PMS to diagnose malignancy was performed and a cut-off value of 14 was determined with the area under the curve (AUC) 0.955 $(p<0.001)$. The sensitivity was $95 \%$, specificity was $80 \%$, positive and negative predictive values were $82.6 \%$ and $94.1 \%$. Likewise, the discriminative value of RMI-3 to diagnose malignancy was evaluated by the ROC curve. AUC for RMI-3 was 0.930 with a sensitivity of $95 \%$, the specificity of $75 \%$, the positive predictive value of $79.1 \%$ and negative predictive value of $93.7 \%$ with a cut-off value of $>53.2(p<0.001)$.

Conclusion: Age, menopause status, tumor markers and sonographic parameters may be beneficial alone for determining malignancy, whereas the scoring systems integrating all the parameters are more powerful. According to our findings, PMS is more sensitive and more practical than the RMI-3 scoring system.

Keywords: Adnexal mass; pelvic mass score; risk of malignancy index.

Please cite this article as "Isgandarova A, Ender Yumru A, Karatas S, Dincgez Cakmak B, Dundar B, Turker UA. The Comparison of Pelvic Mass Score and Risk of Malignancy Index-3 in Discrimination of Benign and Malignant Adnexal Masses. Med Bull Sisli Etfal Hosp 2020;54(4):490-496".
\end{abstract}

Address for correspondence: Burcu Dincgez Cakmak, MD. Saglik Bilimleri Universitesi, Bursa Yuksek Ihtisas Arastirma ve Egitim Hastanesi, Kadin Hastaliklari ve Dogum Klinigi, Bursa, Turkey

Phone: +90 5305448828 E-mail: burcumavis@gmail.com

Submitted Date: November 15, 2018 Accepted Date: January 14, 2019 Available Online Date: December 11, 2020

${ }^{\circ}$ Copyright 2020 by The Medical Bulletin of Sisli Etfal Hospital - Available online at www.sislietfaltip.org

OPEN ACCESS This is an open access article under the CC BY-NC license (http://creativecommons.org/licenses/by-nc/4.0/). 
A dnexa is composed of ovaries, fallopian tubes, broad ligament and the embryologic remnants in the broad ligament. Adnexal masses are quite common among all age groups. It is the fourth most common gynecologic cause of hospital admissions and they are mostly benign. ${ }^{[1]}$ Adnexal masses may have a congenital, functional, inflammatory or neoplastic origin. Despite the important improvements in the diagnosis and treatment opportunities, ovarian cancer is still the most common cause of deaths due to gynecologic malignancies. ${ }^{[2]}$ Since all the adnexal masses have the potential of the malignancy, determination of reliable measurements for follow-up is crucial. Also, when surgical or medical intervention is required choosing the appropriate treatment option promptly is quite important for the prognosis of the patient. ${ }^{[3]}$

Pelvic examination, tumor markers (CA125, CA19-9, CA 15-3, CEA (carcinoembryonic antigen), alpha fetoprotein (AFP), inhibin, HE-4 ve lactate dehydrogenase), ultrasonography, colour Doppler sonography, magnetic resonance imaging (MRI), computerized tomography (CT) and positron emission tomography (PET) are the main modalities of diagnosis for the malignant potential of adnexal masses. ${ }^{[4-6]}$ Since each modality alone does not have enough sensitivity for the discrimination of malignant adnexal masses, the combination of all methods called multimodal screening is being used currently.

In 1990, Jacobs et al. ${ }^{[7]}$ offered using the risk of malignancy index (RMI), which is calculated using serum CA-125 levels, menopause status of women and ultrasonographic findings. According to the calculated risk index, patients were divided into three risk groups as low, moderate and high risk of malignancy and appropriate surgical treatment for each patient was being planned.

In recent years, a new scoring system to investigate the risk of malignancy for pelvic masses, called Pelvic Mass Score (PMS), has been developed. Serum CA-125 levels, the menopausal status of patients, the ultrasonographic features of an adnexal mass, the Sassone score, the vascularity and the resistance index (RI) of adnexal masses have been considered in PMS. ${ }^{[8]}$

In this study, we aimed to compare PMS and RMI-3 scoring systems in determining the malignant potential of adnexal masses.

\section{Methods}

In this study, 40 patients between the age of 15-79 and who were diagnosed as adnexal mass and operated in our gynecology clinic were included. It was conducted as a prospective observational study between the dates of March 2016 and October 2016 at a university-affiliated research and training hospital. Patients were classified as benign $(n=20)$ and malignant $(n=20)$. This study complied with the Declaration of Helsinki, and it was approved by the local ethics committee. Written informed consent was obtained from the study participants.

Patients who did not give consent for surgical treatment and the patients who were pregnant were excluded from this study. All the study participants included in this study underwent surgical treatment. When there is suspicion of malignancy, intraoperative pathologic examination of a frozen specimen was performed. In benign adnexal masses, conservative surgical approach was the choice of the treatment, while in case of malignancy, a radical surgical approach, including surgical staging, was preferred. The surgical specimen was sent to the pathology department for histopathological examination.

The age, gravidity, parity, findings of a pelvic examination, medical history, family history, laboratory parameters, sonographic findings, histopathological results and PMS and RMI-3 scoring results of the patients were recorded.

In sonographic evaluation of patients, the scoring system suggested by Jacobs et al. was used. According to this, multilocularity, bilaterality, the presence of solid areas, any finding of intraabdominal metastasis and the presence of abdominal ascites were analyzed and 1 point for each was assigned. At the same time, the criteria offered by Sassone et al, was used, and the wall structure of the mass, the features of the inner wall, the presence and the properties of septa and the echogenicity of mass were evaluated. For sonographic examination, Siemens Acuson X150 and 6.7 Mhz convex abdominal and 5.0 Mhz vaginal probes were used.

Patients who were diagnosed with adnexal mass underwent color flow Doppler sonography. The sonographic examination was performed in the follicular period (cycle day 3-11) in premenopausal women, whereas the sonographic examination was performed at any time in postmenopausal women. When vascularity was determined in color Doppler, pulse Doppler was activated to yield a waveform. Resistance index (RI: peak systolic velocity-end-diastolic velocity/peak systolic velocity: [s-d/s]) was calculated automatically as three sequential similar flow velocity waveforms were observed. If more than one vascular structures were observed, the least RI value was taken into account. $A$ $\mathrm{RI}$ value $<0.45$ was accepted as malignant and the results were compared with histopathological findings. The dimensions of the affected ovaries were measured in coronal and sagittal planes. The volume of ovaries was calculated using the formula: volume $=$ length $\mathrm{x}$ width $\mathrm{x}$ height $\mathrm{x} \pi / 6$. [9] The serum level of CA-125 was studied after the centrifugation of venous blood sample for 10 minutes at 4000 ppm 
with Roche Cobas 602 immunoassay (Roche Diagnostic USA) using electrochemiluminesans technique. The serum CA-125 level $\leq 35 \mathrm{IU} / \mathrm{ml}$ was accepted as normal. The menopause status was accepted as one year of amennorrhea in women who were spontaneously in menopause and in women who underwent hysterectomy, it was accepted as being over the age of 50 .

\section{Calculation of RMI-3}

RMI-3 was calculated using the formula [U] $\times[M] \times[C A-125]$, where $U$ represents the ultrasonography score, $M$ represents menopausal status and CA-125 represents the serum level of CA-125. Ultrasonography score was accepted " 0 " when none of the findings suggested by Jacobs et al. was present, accepted as "1" when one finding was present and " 3 " when 2 and more were present. Menopausal status was scored as "1" for premenopausal cases and " 3 " for postmenopausal ones. The parameters evaluated in sonographic examination suggested by Jacobs et al. ${ }^{[7]}$ were as follows: multilocularity, presence of solid areas, bilaterality, presence of ascites and metastasis. The cut-off for RMI-3 was taken as 200 .

\section{Calculation of PMS}

For calculation of PMS, the sonographic morphologic pattern of an adnexal mass, the colour Doppler flowmetry features, serum CA-125 level, and the menopausal status of the patient were evaluated. The formula used for the calculation of PMS is as follows: ${ }^{[8]}$

Log(CA-125) $\times$ MS $\times$ SASS $\times$ VAS/RI

Log(CA-125): logaritmic value of serum CA-125 concentration (IU/ ml)

MS: Menopausal status (premenopausal=1; postmenopausal=3)

$\mathrm{RI}$ : numeric pelvic mass $\mathrm{RI}$ value

SASS: Sassone Score $(\min$ score $=4$, $\max$ score $=15$ )

VAS: type of vascularization (peripheral $=1$; central/septal=2)

In the present study, the malignancy risk of patients with adnexal masses was determined using both of the indexes and the sensitivity, specificity, positive and negative predictive values of both indexes were compared.

\section{Statistical Analysis}

Statistical analysis was performed using SPSS version 19.0 (IBM Corp. SPSS Statistics for Windows, Armonk, NY: IBM Corp.). Shapiro-Wilk test was used to decide whether the variables were distributed normally or not. Frequency, percentage, median, minimum and maximum values were used for descriptive parameters. Mann-Whitney-U test and
Chi-square tests were used to compare variables between the benign and malign group. Sensitivity, specificity, positive predictive value and negative predictive value of RMI-3 and PMS scores were calculated for previous cut-off values (200 for RMI-3 and 29 for PMS) and new cut-off values were calculated for both scores using ROC analysis. A p-value $<0.05$ was considered as statistically significant.

\section{Results}

The mean age of the patients included in this study was $42.78 \pm 14.23$ years. When patients were analysed in regard of menopausal status, $26(65 \%)$ of them were premenopausal and 14 (35\%) of them were postmenopausal. Among premenopausal patients, the benign mass was detected in 17 (65.4\%) patients and malignant mass was present in nine (34.6\%) of them. The ratio of benign masses in premenopausal patients was statistically significantly high. On the other hand, the ratio of benign masses was $21.4 \%$ and it was $78.6 \%$ for malignant masses. The malignant masses were significantly common in postmenopausal patients $(p=0.019)$.

The sociodemographic findings of patients and different scoring systems of benign and malignant masses are demonstrated in Table 1. There was no difference between the two groups according to gravida, parity, menarche age, menopause age and ovarian volume. The mean age $(p=0.001)$, serum CA125 levels $(p<0.001)$, Sassone scores $(p=0.001)$ and ultrasonography scores $(p<0.001)$ of ma-

Table 1. Sociodemographic findings of patients and different scoring systems

\begin{tabular}{lccc}
\hline & $\begin{array}{c}\text { Benign Group } \\
(\mathbf{n = 2 0 )}\end{array}$ & $\begin{array}{c}\text { Malign Group } \\
(\mathbf{n = 2 0 )}\end{array}$ & $\mathbf{p}$ \\
\hline Age (years) & $36.1 \pm 11.1$ & $49.55 \pm 14$ & 0.001 \\
Gravida (n) & $2(1-5)$ & $2(1-6)$ & 0.110 \\
Parity (n) & $1(0-5)$ & $1(0-5)$ & 0.080 \\
Menarche (years) & $13.3 \pm 1.22$ & $13.4 \pm 1.19$ & 0.740 \\
Menopause age (years) & $47 \pm 5.66$ & $49.09 \pm 4.09$ & 0.619 \\
Ovarian volume (mm $\left.{ }^{3}\right)$ & $207(190-551)$ & $208(180-462)$ & 0.779 \\
Resistance index & $1.14 \pm 0.31$ & $0.68 \pm 0.16$ & $<0.001$ \\
CA-125 (IU/ml) & $28(5-52)$ & $299(4-519)$ & $<0.001$ \\
PMS score & $9.91 \pm 5.29$ & $55.31 \pm 40.96$ & $<0.001$ \\
RMI-3 score & $36.27 \pm 31.01$ & $1728.14 \pm 325.3$ & $<0.001$ \\
Sassone score & $7.8 \pm 3.19$ & $11.25 \pm 2.22$ & $<0.001$ \\
Ultrasonography score & & & \\
\multicolumn{1}{l}{0} & $9(45 \%)$ & $0(0 \%)$ & $<0.001$ \\
1 & $7(35 \%)$ & $2(10 \%)$ & \\
3 & $4(20 \%)$ & $18(90 \%)$ & \\
\hline
\end{tabular}

PMS: Pelvic mass score; RMI: risk of malignancy index. 
lignant cases were significantly higher than benign cases, whereas the resistance index was lower in malignant group $(p<0.001)$. Moreover, RMI-3 and PMS scores were compared. Both RMI-3 and PMS scores were significantly higher in the malignant group (1728.14 \pm 325.3 vs. $36.27 \pm 31.01, p<0.001$ and $55.31 \pm 40.96$ vs. $9.91 \pm 5.29, p<0.001$, respectively).

When patients were analysed according to the sonographic features used in scoring systems, there was no difference between two groups concerning multilocularity (20\% vs. $45 \% ; p=0.176)$ and bilaterality ( $20 \%$ vs. $40 \% ; p=0.301)$. However, sonographic features which can be sorted as presence of solid areas ( $35 \%$ vs. $90 \% ; \mathrm{p}=0.001$ ), ascites ( $10 \%$ vs. $70 \% ; p<0.001)$, metastasis ( $0 \%$ vs. $35 \% ; p=0.008)$, peripheral blood flow in Doppler flowmetry (40\% vs. $90 \%$; $\mathrm{p}<0.001)$ were more commonly observed in malignant group. In the benign group, $65 \%$ of masses had regular wall structure. In $60 \%$, the wall thickness was $<3 \mathrm{~mm}$, in $55 \%$, there was no septa, and in 45\%, mixt sonographic patern was observed. On the other hand, in the malign group 60\% of masses had solid nodularities in the wall structure, in $60 \%$, there was septation, and in $80 \%$, mixt sonographic patern was present.

The receiver operating curve (ROC) for the predictive value of PMS to diagnose malignancy was performed, and a cut-off value of 14 was determined with AUC 0.955 $(p<0.001)$. The sensitivity was $95 \%$, specificity was $80 \%$, positive and negative predictive values were $82.6 \%$ and 94.1\%. Likewise, the discriminative value of RMI-3 to diagnose malignancy was evaluated by the ROC curve. The area under the curve (AUC) for RMI- 3 was 0.930 with a sensitivity of $95 \%$, the specificity of $75 \%$, the positive predictive value of $79.1 \%$ and negative predictive value of $93.7 \%$ with a cut-off value of $>53.2(p<0.001)$ (Fig. 1$)$. We calculated the sensitivity for RMI-3 scoring system as $60 \%$, specificity as $100 \%$, positive predictive value as $100 \%$ and negative predictive value as $71.4 \%$ when the cut-off point was accepted as 200. The comparison of RMI-3 and PMS scores were demonstrated in Table 2 . In addition, we accepted the cut-off point for PMS as 29 and the sensitivity, specificity, positive predictive value and negative predictive value were calculated as $70 \%, 100 \%, 100 \%$ and $76.9 \%$, respectively.

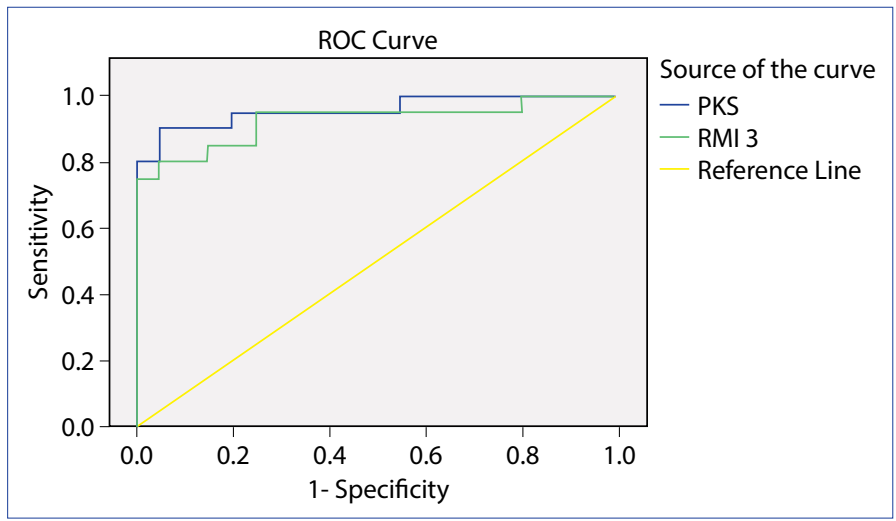

Figure 1. Receiver operating curve (ROC) for the predictive value of PMS and RMI-3 to diagnose malignancy.

\section{Discussion}

The accurate pre-operative evaluation of malignancy potentials of adnexal masses is quite important for the decision of optimum surgical treatment modality, which will provide either the change of cure or disease-free survival. However, most of the time the adnexal masses are diagnosed by intraoperative and/or postoperative histopathological evaluation because of the inadequacy of current diagnostic tools.

Ultrasonography is an important diagnostic tool for the determination of the malignant potentials of adnexal masses. Several scoring systems were developed according to the morphologic characteristics of benign and malignant adnexal masses in transvaginal ultrasonographic examination. ${ }^{[10,11]}$ Although there are little differences between those scoring systems, the size of the mass, the echogenicity, the presence of septa and/or papillary projections, the thickness of the cyst wall and the presence of ascites are generally identical.

The potential role of morphologic features of adnexal masses in the determination of malignant potential is investigated in a study, including 312 patients, and the sensitivity was shown to be $96.8 \%$, specificity was shown to be $77 \%$, the positive predictive value was shown to be $29.4 \%$ and the negative predictive value was $99.6 \% \cdot{ }^{[12]}$ In another study comparing five different sonographic scoring systems (Di Priest, Granberg, Sassone, Lerner, Ferrazzi), the Lerner scoring system displayed the lowest accuracy rate

Table 2. Comparison of RMI-3 and PMS scoring systems

\begin{tabular}{lcccc}
\hline & Sensitivity & Specificity & Positive predictive value & Negative predictive value \\
\hline RMI-3 index (cut-off value 200) & 60 & 100 & 100 & 71.4 \\
PMS index (cut-off value 14) & 95 & 80 & 82.6 & 94.1 \\
\hline
\end{tabular}

PMS: Pelvic mass score; RMI: risk of malignancy index. 
for the discrimination of benign and malignant adnexal masses (57.48\%). On the other hand, the Di Priest scoring system had the highest accuracy rates for the discrimination of benign and malignant adnexal masses (77.16\%). The sensitivity and the false positivity rates for the Di Priest scoring system were shown to be $89.47 \%$ and $21.25 \%$, respectively. ${ }^{[13,14]}$

In our study, Sassone and RMI scoring systems were used. The sensitivity of the Sassone scoring system was reported as $78 \%$ and the specificity was $77 \%$. In accordance with the literature, we demonstrated that the simple cysts had a very low potential of malignancy, while $64 \%$ of complex cysts were observed to be malignant. Considering this, anechogenicity could be accepted as a feature of benign adnexal masses and complex echogenicity as a feature of malignant adnexal masses. In addition to ultrasonographic evaluation, Doppler sonography has an important role in the diagnosis of benign and malignant adnexal masses. Since malignant tumors do not have muscle layer in their vascular structures, they have low impedance and high diastolic flow waves. Thus, many investigators showed that benign and malignant tumors have different hemodynamic features, which could be very useful in early diagnosis of ovarian malignancies using Doppler sonography. ${ }^{[15,16]}$ For that purpose, resistance and pulsatility indexes have been used. ${ }^{[17]}$ In a case series, including 14317 patients, Kurjak et al. ${ }^{[18]}$ reported only two false-negative and one falsepositive results. They accepted 0.40 as a cut-off point for RI. We found statistically significant accordance between the histopathological evaluation and results of RI in Doppler findings. In another study in which the cut-off point for RI was accepted as 0.40 , the sensitivity was $25 \%$ and the specificity was $89 \%{ }^{[19]}$ In the literature, there are several studies that are accepting different cut-off points for RI maintained that it is difficult to standardize Doppler flowmetry analysis. Therefore, it is plausible to state that defining a broadly accepted cut-off point for RI may not be eligible. In the present study, we demonstrated blood flow inside the adnexal mass was in $67.5 \%$ of cases. Among those in which colour flow was not demonstrated, in only one patient, malignancy was confirmed by histopathologic examination. Almost all the malignant adnexal masses color blood flow was observed and their mean values of RI were statistically significantly lower than that of benign masses.

CA-125 has been shown to be the best marker for the clinical follow-up of patients with ovarian cancer. Einhorn et al. showed that its specificity is $98.5 \%$ for cut-off serum level of $\square 35 \mathrm{IU} / \mathrm{ml} .^{[20]}$ Measurement of serum CA-125 is beneficial in discrimination of benign and malignant ovarian masses, in the diagnosis of postmenopausal ovarian masses, in the follow-up of ovarian cancer and in determination of recur- rences following the surgical treatment of ovarian cancer.

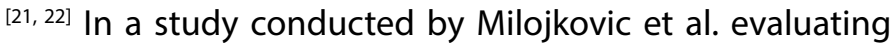
121 ovarian cancer and 91 benign adnexal masses, the sensitivity, specificity, positive predictive value and negative predictive value were $80.2 \%, 76.1 \%, 81.5 \%$ and $74.5 \%$ for cut-off serum level of $\square 35 \mathrm{IU} / \mathrm{ml}$, respectively. Moreover, the sensitivity, specificity, positive predictive value and negative predictive value were $72.7 \%, 90.2 \%, 90.7 \%$ and $71.6 \%$ for cut-off serum level of $\square 65 \mathrm{IU} / \mathrm{ml} .^{[23]}$ In a review, including 17 studies, the sensitivity of CA-125 was reported as $80 \%$ and the specificity was $75 \%$ when the cut-off point was accepted as $35 \mathrm{IU} / \mathrm{ml} .^{[24]}$ In our study, the sensitivity was $70 \%$, specificity was $80 \%$, positive predictive value was $77.8 \%$, and a negative predictive value was $72.7 \%$. However, elevated levels of serum CA- 125 could be seen in several other clinical entities, other scoring systems have been developed taking into account not only serum CA-125 levels but also the morphologic features of mass, the age of patients and the menopausal status.

RMI-3 is one of the scoring system used in the determination of malignancy potential of adnexal masses. RMI-3 developed by Jacobs et al. according to the results of an investigation evaluating 143 patients with adnexal mass and they stated its sensitivity as $85.4 \%$ and specificity as $96.9 \%$. ${ }^{[7]}$ Tingulstad et al. used the revised version of RMI- 3 and they found similar results with Jacobs et al. ${ }^{[25]}$ In most of the studies the cut-off point for determination of malignancy with RMI-3 scoring system was accepted as $200 .{ }^{[7,26-28]}$ In the study of Obeidat et al. ${ }^{[29]}$ including 100 patient with ovarian tumors, the sensitivity was $90 \%$ and the specificity was $89 \%$, positive predictive value was $96 \%$ and negative predictive value was $78 \%$ when the cut-off point for RMI-3 was accepted as 200. In another study using same cut-off value, its sensitivity was reported to be $70.6 \%$, specificity was $87.7 \%$, positive predictive value was $66.1 \%$ and negative predictive value was $89.8 \%$. ${ }^{[30]}$

Morgante et al. ${ }^{[31]}$ investigated the sensitivity, specificity, positive predictive value and negative predictive value of RMI-3 in 124 patients and their results were as follows $58 \%$, $95 \%, 78 \%$ and $87 \%$, respectively. Consistent with the literature, we calculated the sensitivity for RMI-3 scoring system as $60 \%$, specificity as $100 \%$, positive predictive value as $100 \%$, and negative predictive value as $71.4 \%$ when the cut-off point was accepted as 200. Additionally, we defined a new cut-off point for the prediction of malignancy in adnexal masses. According to the ROC analysis when 53.2 is taken as cut-off point for RMI-3 a sensitivity of $95 \%$, a specificity of $75 \%$, a positive predictive value of $79.1 \%$ and a negative predictive value of $93.7 \%$ were calculated. Consequently, we think that patients with RMl-3 score $>53.2$ must be managed much more carefully in regard of malignancy. 
The logical reason for lower cut-off point that we calculated may be the number of patients included in this study and/or the similarity between the number of patients who have malignant adnexal mass in both premenopausal and postmenopausal groups.

Another scale used for the evaluation of malignant potential of adnexal masses is PMS. When the cut-off point was accepted as 29 , its sensitivity was observed to be $100 \%$, specificity was $93.8 \%$, positive predictive value was $70 \%$ and negative predictive value was $100 \%$ by Rossi et al. ${ }^{[8]}$ In another study using the same cut-off value for PMS scoring system, the sensitivity was $92.9 \%$, specificity was $80.2 \%$, positive predictive value was $75.6 \%$ and negative predictive value was $94.4 \% \cdot{ }^{[32]}$ In the present study, we accepted the cut-off point for PMS as 29 and the sensitivity, specificity, positive predictive value and negative predictive value were calculated as $70 \%, 100 \%, 100 \%$ and $76.9 \%$, respectively. The data about the PMS scoring system in the literature are limited. When we take the cut-off point for PMS as 14 in our study, the sensitivity was calculated as $95 \%$, specificity as $80 \%$, positive predictive value as $82.6 \%$ and negative predictive value as $94.1 \%$.

Considering the results of the present study, it is plausible to infer that using PMS scoring sytem may improve the diagnostic success of adnexal masses. It was shown that PMS has a high discrimination capacity to select histopathologically malignant cases among preoperative malignant ones (95\%), whereas it has a low capacity to choose histopathologically benign ones among preoperative benign cases (80\%). Unlike Rossi et al., the cut-off point for PMS scoring system 14 was taken instead of 29 in our study that it could be related to ratios of malignant/benign patients.

\section{Conclusion}

Age, menopause status, tumor markers and sonographic parameters may be beneficial for discriminating malignant adnexal masses from benign ones separately. However, the scoring sytems integrating all the above parameters are much more powerful in determining malignant potential of adnexal masses. According to our findings, PMS is more sensitive and more practical than the RMI-3 scoring system. However, to make an accurate conclusion, prospective studies with larger sample sizes are required to compare these scoring systems.

\section{Disclosures}

Ethics Committee Approval: The study was approved by Sisli Hamidiye Etfal Research and Training Hospital, Clinical Research Ethics Committee with a number of 639 at the date of 12/04/2016.

Peer-review: Externally peer-reviewed.

\section{Conflict of Interest: None declared.}

Authorship Contributions: Concept - A.I., A.E.Y.; Design - A.I, S.K.; Supervision - B.D.C., B.D., A.E.Y.; Materials - A.I., S.K.; Data collection \&/or processing - A.I., S.K.; Analysis and/or interpretation - U.A.T., B.D., B.D.C.; Literature search - U.A.T.; Writing - U.A.T., B.D., B.D.C.; Critical review - A.E.Y.

\section{References}

1. Sık BA, Erdem B, Aba YA, Kumbasar S. Adnexal masses: Clinical approach and pathological findings. Journal of Human Sciences 2016;13:2870-8. [CrossRef]

2. Holschneider $\mathrm{CH}$, Berek JS. Ovarian cancer: epidemiology, biology, and prognostic factors. Semin Surg Oncol 2000;19:3-10.

3. Westhoff $C$, Randall MC. Ovarian cancer screening: potential effect on mortality. Am J Obstet Gynecol 1991;165:502-5. [CrossRef]

4. Stein SM, Laifer-Narin S, Johnson MB, Roman LD, Muderspach LI, Tyszka JM, et al. Differentiation of benign and malignant adnexal masses: relative value of gray-scale, color Doppler, and spectral Doppler sonography. AJR Am J Roentgenol 1995;164:381-6.

5. Kurtz AB, Tsimikas JV, Tempany CM, Hamper UM, Arger PH, Bree $\mathrm{RL}$, et al. Diagnosis and staging of ovarian cancer: comparative values of Doppler and conventional US, CT, and MR imaging correlated with surgery and histopathologic analysis--report of the Radiology Diagnostic Oncology Group. Radiology 1999;212:1927. [CrossRef]

6. Grab D, Flock F, Stöhr I, Nüssle K, Rieber A, Fenchel S, et al. Classification of asymptomatic adnexal masses by ultrasound, magnetic resonance imaging, and positron emission tomography. Gynecol Oncol 2000;77:454-9. [CrossRef]

7. Jacobs I, Oram D, Fairbanks J, Turner J, Frost C, Grudzinskas JG. A risk of malignancy index incorporating CA 125, ultrasound and menopausal status for the accurate preoperative diagnosis of ovarian cancer. Br J Obstet Gynaecol 1990;97:922-9. [CrossRef]

8. Rossi A, Braghin C, Soldano F, Isola M, Capodicasa V, Londero AP, et al. A proposal for a new scoring system to evaluate pelvic masses: Pelvic Masses Score (PMS). Eur J Obstet Gynecol Reprod Biol 2011;157:84-8. [CrossRef]

9. Pavlik EJ, DePriest PD, Gallion HH, Ueland FR, Reedy MB, Kryscio RJ, et al. Ovarian volume related to age. Gynecol Oncol 2000;77:4102. [CrossRef]

10. Lerner JP, Timor-Tritsch IE, Federman A, Abramovich G. Transvaginal ultrasonographic characterization of ovarian masses with an improved, weighted scoring system. Am J Obstet Gynecol 1994;170:81-5. [CrossRef]

11. Ionescu CA, Matei A, Navolan D, Dimitriu M, Bohâltea R, Neacsu $A$, et al. Correlation of ultrasound features and the Risk of Ovarian Malignancy Algorithm score for different histopathological subtypes of benign adnexal masses. Medicine (Baltimore) 2018;97:e11762. [CrossRef]

12. Sassone AM, Timor-Tritsch IE, Artner A, Westhoff C, Warren WB. Transvaginal sonographic characterization of ovarian disease: 
evaluation of a new scoring system to predict ovarian malignancy. Obstet Gynecol 1991;78:70-6.

13. Gramellini D, Rutolo S, Verrotti C, Piantelli G, Fieni S, Vadora E. Sonographic characterization, Doppler ultrasonography and tumor markers in the diagnosis of malignancy of ovarian masses. Minerva Ginecol 2001;53:1-11.

14. Greenlee RT, Hill-Harmon MB, Murray T, Thun M. Cancer statistics, 2001. CA Cancer J Clin 2001;51:15-36. [CrossRef]

15. Maly Z, Riss P, Deutinger J. Localization of blood vessels and qualitative assessment of blood flow in ovarian tumors. Obstet Gynecol 1995;85:33-6. [CrossRef]

16. Dai SY, Hata K, Inubashiri E, Kanenishi K, Shiota A, Ohno M, Yamamoto $Y$, Nishiyama Y, Ohkawa M, Hata T. Does three-dimensional power Doppler ultrasound improve the diagnostic accuracy for the prediction of adnexal malignancy? J Obstet Gynaecol Res 2008;34:364-70. [CrossRef]

17. Barroilhet L, Vitonis A, Shipp T, Muto M, Benacerraf B. Sonographic predictors of ovarian malignancy. J Clin Ultrasound 2013;41:26974. [CrossRef]

18. Kurjak A, Zalud I, Alfirevic Z. Evaluation of adnexal masses with transvaginal color ultrasound. J Ultrasound Med 1991;10:295-7.

19. Levine D, Feldstein VA, Babcook CJ, Filly RA. Sonography of ovarian masses: poor sensitivity of resistive index for identifying malignant lesions. AJR Am J Roentgenol 1994;162:1355-9. [CrossRef]

20. Einhorn N, Sjövall K, Knapp RC, Hall P, Scully RE, Bast RC Jr, et al. Prospective evaluation of serum CA 125 levels for early detection of ovarian cancer. Obstet Gynecol 1992;80:14-8.

21. Guppy AE, Rustin GJ. CA125 response: can it replace the traditional response criteria in ovarian cancer? Oncologist 2002;7:437-43.

22. Meyer T, Rustin GJ. Role of tumour markers in monitoring epithelial ovarian cancer. Br J Cancer 2000;82:1535-8.

23. Milojkovic M, Hrgovic Z, Hrgovic I, Jonat W, Maass N, Buković D. Significance of CA 125 serum level in discrimination between benign and malignant masses in the pelvis. Arch Gynecol Obstet 2004;269:176-80. [CrossRef]

24. Medeiros LR, Rosa DD, da Rosa MI, Bozzetti MC. Accuracy of CA 125 in the diagnosis of ovarian tumors: a quantitative systematic review. Eur J Obstet Gynecol Reprod Biol 2009;142:99-105.

25. Tingulstad S, Hagen B, Skjeldestad FE, Onsrud M, Kiserud T, Halvorsen $T$, et al. Evaluation of a risk of malignancy index based on serum CA125, ultrasound findings and menopausal status in the pre-operative diagnosis of pelvic masses. Br J Obstet Gynaecol 1996;103:826-31. [CrossRef]

26. Tingulstad S, Hagen B, Skjeldestad FE, Halvorsen T, Nustad K, Onsrud $M$. The risk-of-malignancy index to evaluate potential ovarian cancers in local hospitals. Obstet Gynecol 1999;93:448-52.

27. Manjunath AP, Pratapkumar, Sujatha K, Vani R. Comparison of three risk of malignancy indices in evaluation of pelvic masses. Gynecol Oncol 2001;81:225-9. [CrossRef]

28. Ma S, Shen K, Lang J. A risk of malignancy index in preoperative diagnosis of ovarian cancer. Chin Med J (Engl) 2003;116:396-9.

29. Obeidat BR, Amarin ZO, Latimer JA, Crawford RA. Risk of malignancy index in the preoperative evaluation of pelvic masses. Int J Gynaecol Obstet 2004;85:255-8. [CrossRef]

30. Andersen ES, Knudsen A, Rix P, Johansen B. Risk of malignancy index in the preoperative evaluation of patients with adnexal masses. Gynecol Oncol 2003;90:109-12. [CrossRef]

31. Morgante G, la Marca A, Ditto A, De Leo V. Comparison of two malignancy risk indices based on serum $C A 125$, ultrasound score and menopausal status in the diagnosis of ovarian masses. $\mathrm{Br} \mathrm{J}$ Obstet Gynaecol 1999;106:524-7. [CrossRef]

32. Ibrahim M, Hanafi S, Ibrahim A, El-Garhy R, AkI S. Validity of the pelvic mass score in comparison with other scoringsystems in preoperative evaluation of ovarian masses. Evidence Based Women's Health Journal 2014;4:127-34. [CrossRef] 